\title{
Loss of engagement in controlling chronic anticoagulation therapy during COVID-19 stringency measures. A single center experience of disproportioned increase of stuck mechanical valves
}

\author{
Olga Vriz ${ }^{1,2}$, Andrea Rossi Zadra ${ }^{1}$, Abdalla Eltayeb ${ }^{1}$, Fatima Asiri ${ }^{1}$, Claudio Pragliola ${ }^{3}$, \\ Nader Ashraf Fawzy ${ }^{2}$, Domenico Galzerano', Khalid Feras' ${ }^{1}$, Zohair Alhalees ${ }^{1}$, Abdulhalim J Kinsara ${ }^{4}$, \\ Fadl EImula Mohamed Fadl Elmula ${ }^{1}$
}

${ }^{1}$ Heart Centre Department, King Faisal Specialist Hospital and Research Center, Riyadh; ${ }^{2}$ College of Medicine, Alfaisal University, Riyadh; ${ }^{3}$ Department of Adult Cardiac Surgery, Prince Sultan Cardiac Center, Riyadh; ${ }^{4}$ Ministry of National Guard Health Affair, COM-WR, King Abdullah International Research, Saudi Arabia

\begin{abstract}
Stuck valve is a very rare and severe complication that occurs in mechanical valve replacement patients with ineffective anticoagulation. However, with COVID-19 restriction measures, it became challenging to regularly assess INR to make sure it falls
\end{abstract}

Correspondence: Prof Olga Vriz, Heart Centre, King Faisal Specialist Hospital and Research Centre, Zahrawi St, Al Maather, Al Maazer, Riyadh 12713, Saudi Arabia.

Tel. +966.1.1464-7272 extension 32056

Fax: +966.1.14427791.

E-mail: olgavriz@yahoo.com

Key words: stuck valve; cardiology; chronic anticoagulant.

Contributions: All authors contributed equally to the paper, read and approved the final version of the manuscript and agreed to be accountable for all aspects of the work.

Conflict of interest: The authors declare that they have no competing interests, and all authors confirm accuracy.

Ethics Approval: The study protocol approved by the Ethics Committee at King Faisal Hospital has the following identification: RAC \# 2201091: COVID-19 and Cardiovascular Diseases.

Informed Consent: The informed consent from patients has been waived by the Ethics Committee of our institute.

Funding: This research received no specific grant from any funding agency in the public, commercial, or not-for-profit sectors.

Received for publication: 25 August 2021.

Accepted for publication: 22 September 2021.

${ }^{\circ}$ Copyright: the Author(s), 2021

Licensee PAGEPress, Italy

Monaldi Archives for Chest Disease 2022; 92:2065

doi: 10.4081/monaldi.2021.2065

This article is distributed under the terms of the Creative Commons Attribution Noncommercial License (by-nc 4.0) which permits any noncommercial use, distribution, and reproduction in any medium, provided the original author(s) and source are credited. within the target therapeutic range to prevent this complication. We present a series of 10 patients who either underwent transthoracic echocardiography for a suspected stuck valve or were seen at the outpatient valve clinic with the residual consequences of a stuck valve during the COVID-19 restriction measures in our institute. Stuck prosthetic valves incident has increased significantly during this period, particularly those in the mitral position for which urgent replacement and prolonged hospitalization were necessary. Particularly with the COVID-19 restrictions in place, these cases highlight the need for physicians to be aware of the dramatic increase in the incidence of stuck prosthetic valves in patients on chronic warfarin therapy.

\section{Introduction}

In December 2019, coronavirus disease (COVID-19) emerged in Wuhan, Hubei Province, China, and the WHO raised its classification from 'epidemic' to 'pandemic' on March 11 2020 [1]. In response, hospitals had to both increase their surge capacities in emergency and intensive care departments but also significantly reduce internal medicine, family medicine, routine out-patient clinic and laboratory check-up activities [2,3] As such, several countries prohibited non-essential surgical and oncological elective procedures for an indeterminate period of time [4,5] As yet, it is unclear how the duration, timing and nature of these measures impacted patients, and the long-term consequences of limiting their access to health services, including simple routine coagulation screening, is yet to be seen. During the pandemic, patient access to healthcare was further impeded by quarantine measures and curfews, and compounded by elevated numbers of missed appointments due to nervous patients choosing to avoid hospital settings.

During the COVID-19 pandemic, Saudi Arabia, like most countries worldwide, imposed restrictions on people's movements. As such, regular outpatient follow-ups were performed remotely by telephone, and patients were only occasionally requested to attend clinic in-person where necessary for routine laboratory checks, such as determining international normalized ratio (INR).

Warfarin, a vitamin $\mathrm{K}$ inhibitor, is used in patients with mechanical valve prosthesis to prevent thromboembolic events and stuck valves, among other indications. In mechanical valves, a target INR range of 2.5-3.5 is the current recommendation for mechanical 
mitral valve replacement. Regular assessment of INR is necessary to maintain its value within the target therapeutic range.

Herein, we describe a case series of patients on warfarin anticoagulation therapy with stuck mechanical valves during the COVID-19 restriction measures in Saudi Arabia.

\section{Case series}

This is a single-center prospective study of consecutive patients seen in our clinic between January 2000 - January 2021 who either i) underwent transthoracic echocardiography for a suspected stuck valve or ii) were seen at the outpatient valve clinic with the residual consequences of a stuck valve. During this period, 10 patients (mean age $53.9 \pm 16.2$ years, $80 \%$ female) were admitted to the King Faisal Specialist Hospital and Research Center, tertiary referral hospital in Saudi Arabia, for further diagnosis and management of a suspected stuck cardiac valve.

Diagnosis of stuck valve was confirmed in 9/10 patients, and one patient was diagnosed with the residual effects of stroke. For this patient, diagnosis was performed at the local hospital where the patient received medical treatment (Table 1).

All patients were COVID-19 negative; general characteristics are summarized in Table 1. Five patients $(50 \%)$ were from outside Riyadh city, four patients were admitted through the emergency department (Figure 1) and two were referred from other hospitals. In two cases, stuck valve was an incidental finding observed during a routine echocardiographic follow-up, and patients did not complain of specific symptoms.

Eight patients were female, two of whom were pregnant at the first trimester and eventually went on to receive an abortion; six patients received urgent valve replacement cardiac surgery; four patients were managed medically e.g., with heparin; none had thrombolytic therapy. In two patients, surgery was contraindicated by comorbidities and severely compromised left ventricular function (marked by an ejection fraction $<25 \%$ ). Four patients died, including one pregnant woman following surgical valve replacement and maximal support on extracorporeal support (ECMO) over 36 days of hospitalization. In three cases, patients clearly reported that they were afraid to attend the hospital for determination of their INR. The diagnosis of stuck valve was performed by transthoracic echocardiography and transesophageal echocardiography and fluoroscopy was done in $80 \%$ of the patients (Figure 2). Mean hospital admission time was $27.5 \pm 18.5$ days. The cost of treatment, as average estimated reimbursement, was 11,000 USD for medical admission only, and 43,200 USD when surgery was required, up to 64,000 USD when ECMO was part of patient management. As a comparison, from 2015-2019 only one patient per year was admitted to our institute with stuck valve, of whom two died during hospitalization due to complications (Figure 3).

\section{Discussion}

Shortly after the beginning of the COVID-19 pandemic, concerns were raised about the potential negative consequences resulting from limiting elective or non-Covid healthcare services [6], as the number of follow-up and primary care services dramatically decreased due to concerns about virus transmission. These concerns were shared by both patients and healthcare providers, particularly those working in outpatient settings [7].
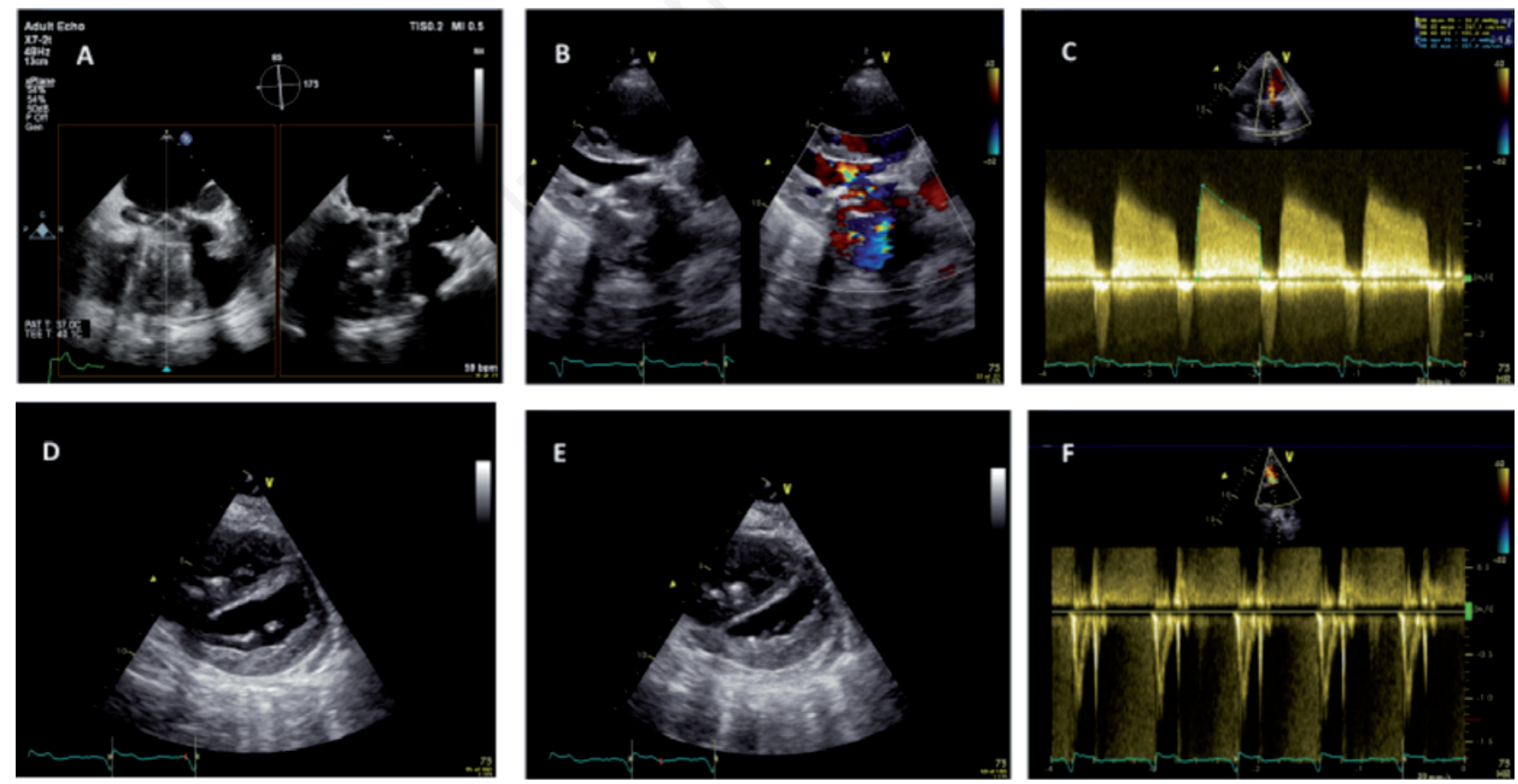

Figure 1. The case of emergency admission of a 36 years old pregnant woman. A) TEE with disks closed in diastole. B) TTE parasternal long axis in diastole. C) CW across the valve, peak and mean gradients $46 / 26 \mathrm{mmHg}$ at $75 \mathrm{bpm}$. D,F) Short axis with $\mathrm{D}$ shape of the left ventricle suggestive of right pressure/volume overload. F) Pulse Doppler on the pulmonary valve with very short acceleration time suggestive of significant increase in pulmonary pressure. 


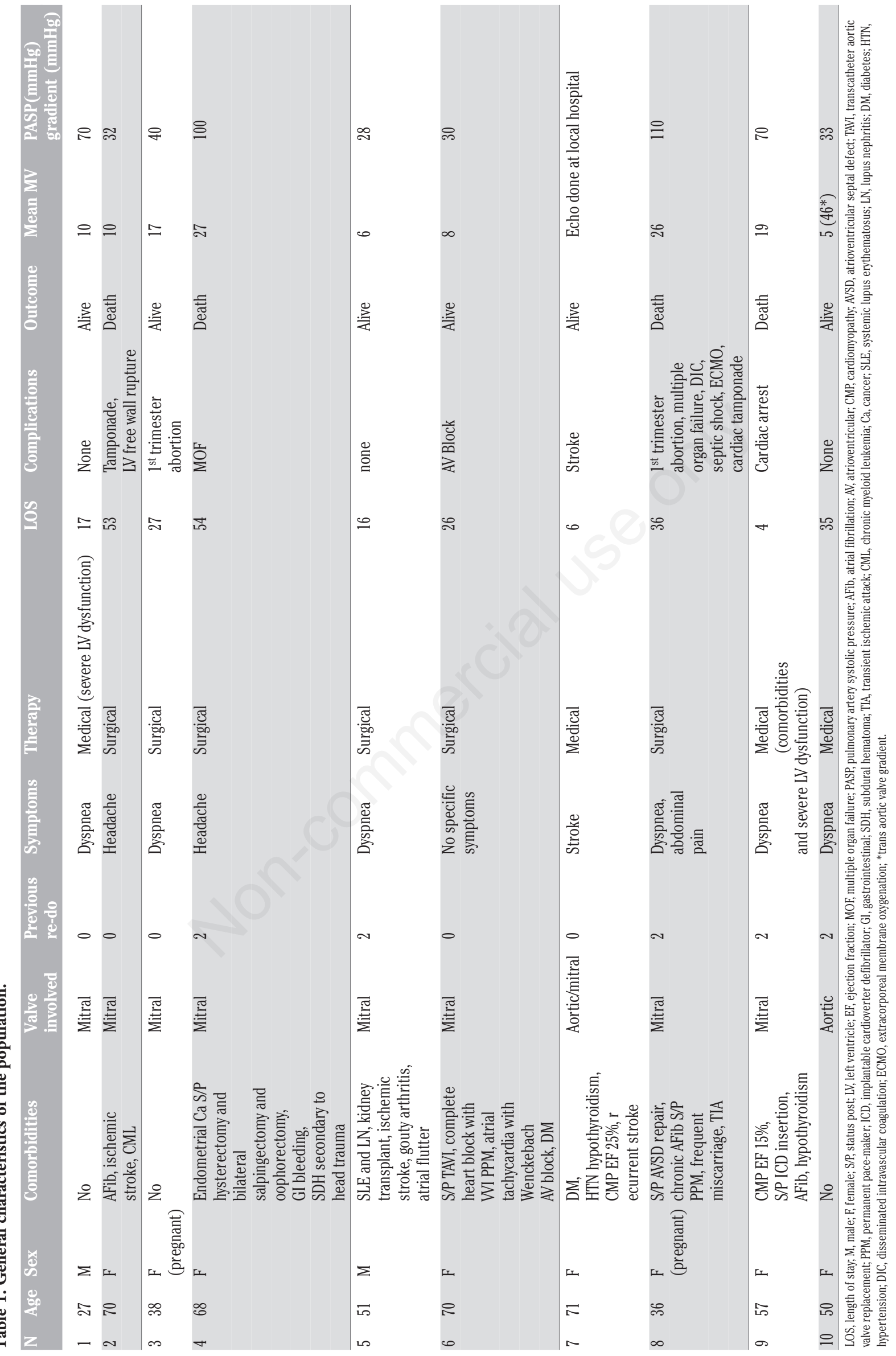




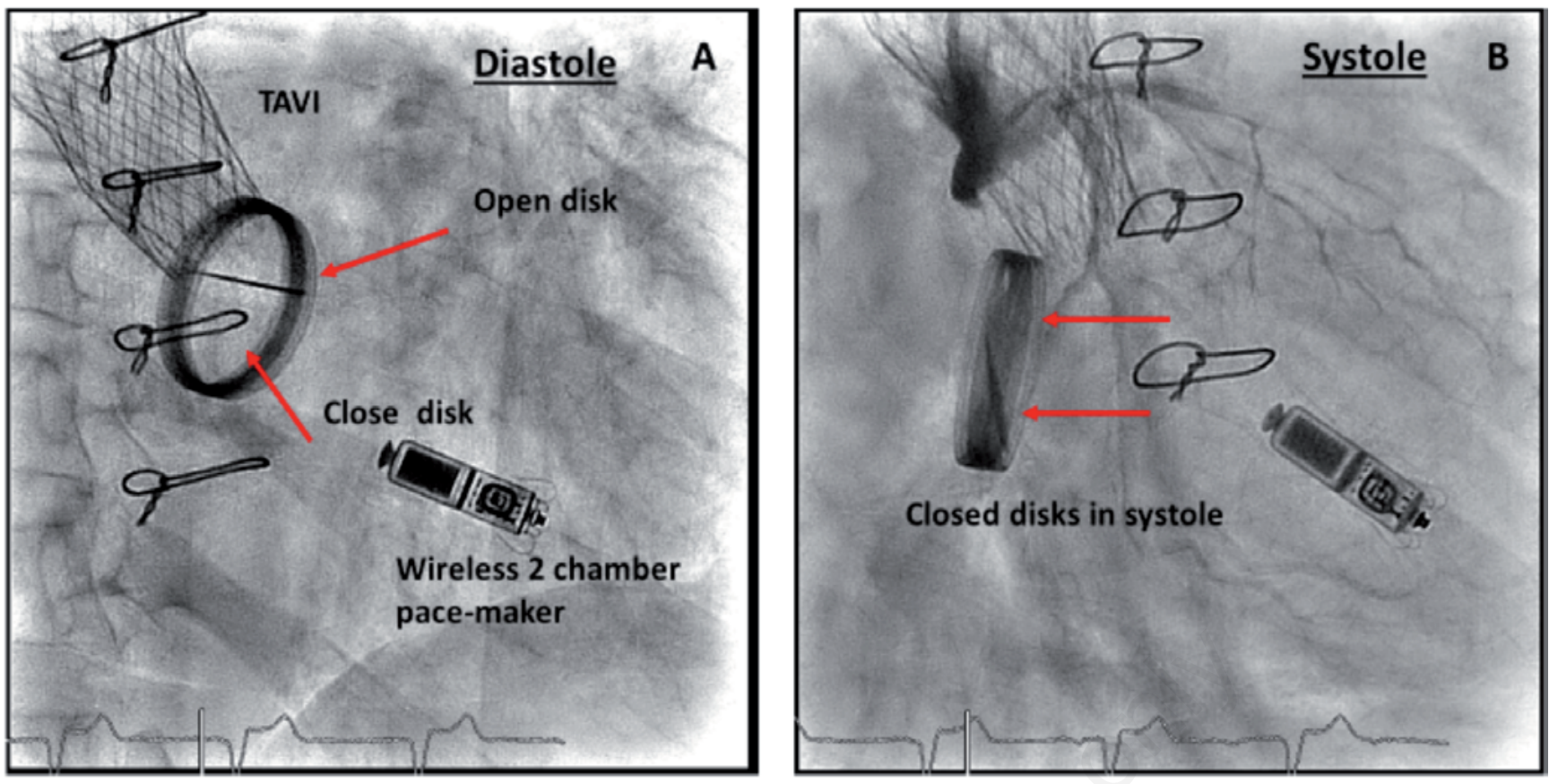

Figure 2. Fluoroscopy in a 70 years old patient. A) Diastole: red arrow address one closed and one open disk. B) Systole: both disks are closed.

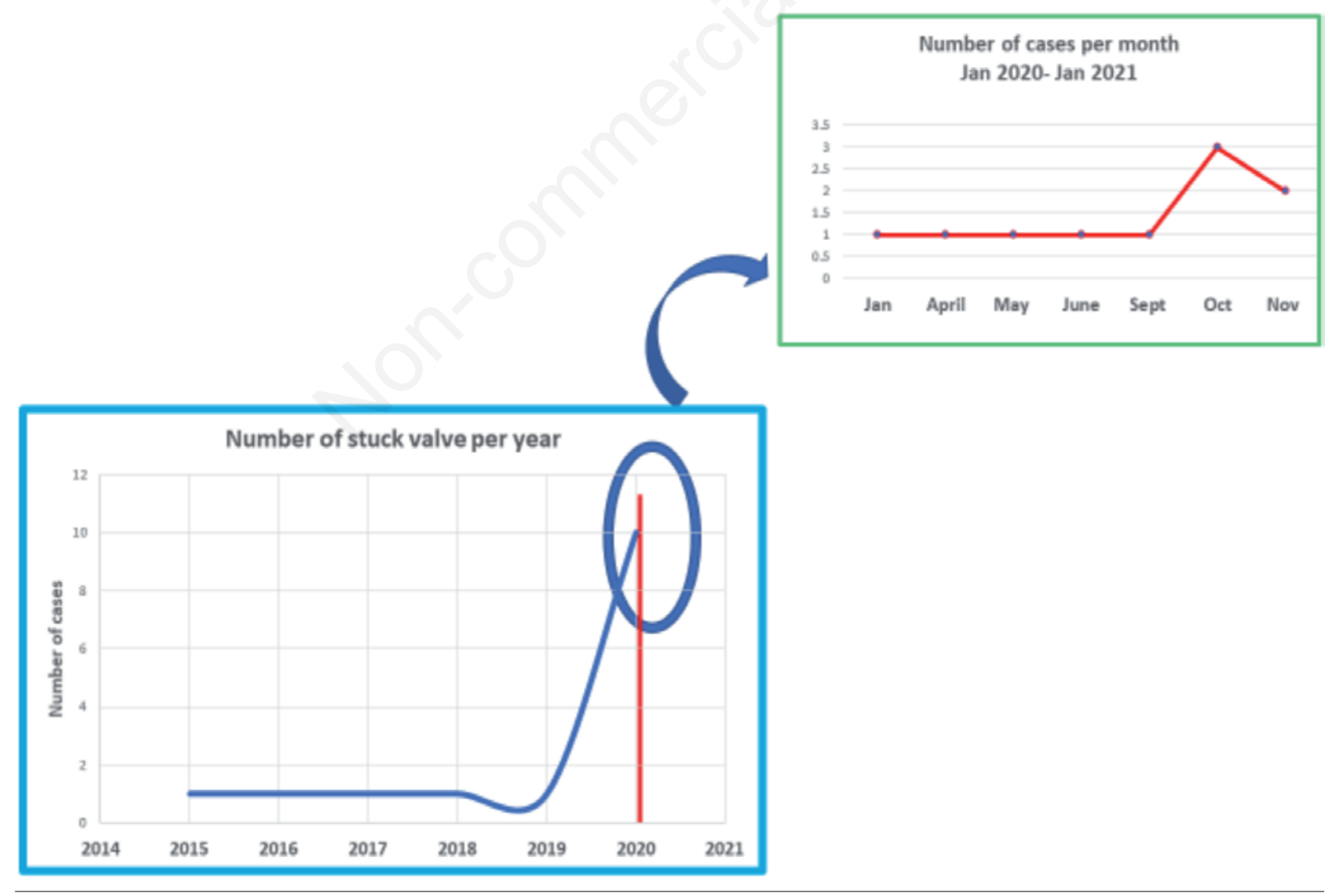

Figure 3. The average number of patients with a mechanical stuck valve is constant over the timeframe 2015-2019, with an increase in the last half of 2020 . 
As a consequence, hospitals deferred elective and preventive consultations, such as annual physicals, and implemented telemedicine services where possible. Moreover, many patients cancelled their scheduled appointments to limit their risk exposure by staying at home. These behaviors were reinforced by local and government recommendations restricting travel and nonessential services statewide.

In our institution, between March and August 2020, outpatient clinics were run by telephone assessment, and the echocardiography laboratory was available for emergencies only. As such, direct face-to-face interaction was not possible, limiting the normal exchange of information between doctor and patient typically expected from a full consultation. Similarly, many patients on warfarin medication did not regularly attend the anticoagulation clinics -despite these services remaining open- likely resulting in a high rate of patients missing their INR target ranges.

We report a dramatic increase in stuck prosthetic valves during this period, particularly those in the mitral position, which in most cases required urgent replacement and prolonged hospitalization, including intensive care admission, with high in-hospital mortality rates and high costs. Some patients were referred from other hospitals, while others were admitted directly from the emergency department with compromised hemodynamics. Two were identified during regular echocardiographic follow-ups when the outpatient clinics were reopened, corresponding with the increased incidence observed in October-December. While repeat heart valve surgery is generally a high-risk intervention [8], poor outcomes have been reported in patients with severe valvular disease whose valve replacement was deferred for replacement/repair until after pandemic restrictions were lifted [9]. In the particular case of stuck valves, the reported mortality is very high: from $69 \%$ in reports back to $1980-1990$ [10] to $35 \%$ in more recent reports [11]. We were unable to find any data concerning the consequences of curfews associated with the COVID-19 pandemic and routine regular assessment of INR in anticoagulation therapy in patients with mechanical heart valves. In general, as a consequence of the COVID-19 restrictions, substantial reductions have been reported in primary care for acute physical and mental conditions [12]

Of particular note, in a survey of 388 patients from the Cardiology Out-Patient Department of a single center in Saudi Arabia, Samargandy et al. [13] reported that $36.3 \%$ of patients did not seek help due to fear of infection. Of these patients, $15.7 \%$ were concerned about violating curfew, 3.9\% were concerned about travelling / public transportation while $44.1 \%$ were neutral. Of particular note, $69 \%$ of these patients reported that they would not have made the same decision had the pandemic not occurred. Most likely our patients - were unable to receive regular INR assessments - fell into similar categories. From a health systems perspective, consistent resources are necessary to provide hightechnology healthcare to patients with highly predictable complications. The direct cost of avoidable complications that require either medical or surgical intervention vastly exceeds the cost of routine assessment of INR value. An unmeasured consequence of avoidable prolonged admissions is the limitation of the number of available beds for emergency or elective admissions, which will further negatively impact health outcomes in a given population [14], especially during the COVID-19 pandemic.

Also, social factors cannot be excluded. Interestingly, $80 \%$ of patients from our sample were women, and some speculation may be needed to explain this interesting finding. The most common reason for valve replacement in Saudi Arabia is rheumatic disease; while this affects females more than males, social factors cannot be excluded. Health disparities between genders is a well-described, worldwide phenomenon that leads to gender inequalities in terms of education, job opportunities, income, family engagement, and may well also affect outcomes during the COVID-19 pandemic. 15,16 One could speculate that the overwhelming demands and commitments of family during this time of profound uncertainty has led many women to heed less attention to their own personal health.

\section{Conclusions}

Despite government efforts to implement telemedicine and virtual clinics, COVID-19 restrictions and health-messaging affected the routine follow-up of non-COVID-19 patients. Clinics were missed for multifactorial reasons, with personal and social factors at play. We report dramatic consequences to this drastic change in healthcare: clinical complications, lost lives and inflated healthcare costs in patients with a prosthetic valve. We believe that nonCOVID-19 patients have paid, and will continue to pay in the future, a price that is much greater than those patients who have suffered from COVID-19. Active engagement of patients should be implemented in a time of emergencies, and further studies should address the efficacy and cost-effectiveness of public policies aimed to decrease the burden of avoidable complications and missed diagnoses.

\section{References}

1. Wu Z, McGoogan JM. Characteristics of and important lessons from the coronavirus disease 2019 (COVID-19) outbreak in China: Summary of a report of 72314 cases from the Chinese center for disease control and prevention. JAMA 2020; 323:1239.

2. Veldtman GR, Pirisi M, et al. Management principles in patients with COVID-19: perspectives from a growing global experience with emphasis on cardiovascular surveillance. Open Heart 2020;7:e001357.

3. Nash DB, Angelo M, Nash EJ, et al. We know health is not elective: Impacts of COVID-19. Popul Health Manag 2020;23: 378-85.

4. Hübner M, Zingg T, Martin D, et al. Surgery for non-Covid19 patients during the pandemic. PLoS One 2020;15: e0241331.

5. Jazieh AR, Akbulut H, Curigliano G, et al. Impact of the COVID-19 pandemic on cancer care: A global collaborative study. JCO Glob Oncol 2020;6:1428-38.

6. Rosenbaum L. The untold toll - The Pandemic's effects on patients without Covid-19. N Engl J Med 2020;382:2368-71.

7. Shah BN, Schlosshan D, McConkey HZR, et al. Outpatient management of heart valve disease following the COVID-19 pandemic: implications for present and future care. Heart 2020;106:1549-54.

8. Ejiofor JI, Hirji SA, Val FR-D, et al. Outcomes of repeat mitral valve replacement in patients with prior mitral surgery: A benchmark for transcatheter approaches. J Thorac Cardiovasc Surg 2018;156:619-627.e1.

9. Ryffel C, Lanz J, Corpataux N, et al. Mortality, stroke, and hospitalization associated with deferred vs expedited aortic valve replacement in patients referred for symptomatic severe aortic stenosis during the COVID-19 pandemic. JAMA Netw Open 2020;3:e2020402. 
10. Deviri E, Sareli P, Wisenbaugh T, Cronje SL. Obstruction of mechanical heart valve prostheses: Clinical aspects and surgical management. J Am Coll Cardiol 1991;17:646-50.

11. Azpitarte J, Sánchez-Ramos J, Oyonarte JM, et al. [Trombosis valvular protésica: ¿cuál es la terapia inicial más apropiada?].[Article in Spanish]. Rev Esp Cardiol2001;54:1367-76.

12. Mansfield KE, Mathur R, Tazare J, et al. Indirect acute effects of the COVID-19 pandemic on physical and mental health in the UK: a population-based study. Lancet Digit Health 2021;3:e217-30.

13. Samargandy S, Al Garni TA, Almoghairi A, et al. Effect of
COVID-19 pandemic on the cardiac outpatients' perception of seeking medical advice. J Saudi Heart Assoc 2020;32:377-82.

14. Kheirkhah P, Feng Q, Travis LM, et al. Prevalence, predictors and economic consequences of no-shows. BMC Health Serv Res 2016;16:13.

15. Shaw LJ, Pepine CJ, Xie J, et al. Quality and equitable health care gaps for women. J Am Coll Cardiol 2017;70:373-88.

16. OECD [Internet]. Women at the core of the fight against COVID-19 crisis. Available from: https:/www.oecd.org/coronavirus/policy-responses/women-at-the-core-of-the-fightagainst-covid-19-crisis-553a8269/ 